\title{
Effect of food consistency on gastric emptying in man
}

\author{
L A HOUGHTON, F HICKSON, AND N W READ
}

From the Sub-Department of Human Gastrointestinal Physiology and Nutrition, Royal Hallamshire Hospital, Sheffield

SUMMARY The gastric emptying of two radiolabelled pancake meals of identical composition, but different consistency, was compared with the rates at which they could be broken down in vitro by mechanical agitation in a solution of either acid-pepsin or saline. Neither of the pancake meals were broken up within 150 minutes by mechanical agitation in a normal saline medium, but both were disrupted in the presence of acid-pepsin, the light consistency pancake being broken up more rapidly than the heavy consistency pancake $(0 \cdot 5 \pm 0 \cdot 1 v 0 \cdot 2 \pm 0 \cdot 1 \%$ radioactivity appearing in the bathing medium per minute, $\mathrm{p}<0 \cdot 01)$. Under normal conditions, the pattern and rate of gastric emptying of both meals was similar. Stimulating acid secretion with pentagastrin $(6 \mu \mathrm{g} / \mathrm{kg}$ bodyweight sc) delayed the onset of emptying of both pancakes and tended to increase the slope of emptying. Under these conditions, the denser pancake was emptied more slowly than the lighter pancake because of an increase in the lag period. Thus the data did not confirm the hypothesis that the rate of emptying of disruptible solid food from the stomach under normal conditions of acid secretion is influenced by the ease with which it can be broken down by mechanical agitation in an acid-pepsin medium. The trend for an accelerated rate of emptying after administration of pentagastrin, however, may well be explained by the increased rate of disruption of the food in the presence of acid, but the longer lag periods are more compatible with inhibition of gastric emptying caused by free acid in the duodenum.

Solids empty from the stomach more slowly than liquids. ${ }^{-1}$ Small particles of solid food are broken down and empty from the stomach more quickly than large solid particles. ${ }^{25}$ The delay in emptying of solid food has been explained by the time taken for large particles to be broken down by mechanical grinding and peptic digestion into particles small enough to pass through the pylorus, ' as thoroughly triturated solids empty at similar rates to liquids. ${ }^{2}$

The aim of this study was to investigate the hypothesis that the rate of emptying of solid food is directly related to the rate at which it can be disrupted in the presence of gastric secretions by comparing the rate of emptying of two radiolabelled solid meals of identical composition; one which was easily disrupted by mechanical agitation in acid-pepsin mixture, the other which took much longer to break down.

Address for correspondence: Dr N W Read, Sub-Department of Human Gastrointestinal. Physiology and Nutrition, K Floor, Royal Hallamshire Hospital. Sheffield S10 2JF.

Received for publication 21 May 1987

\section{Methods}

PREPARATION OF RADIOLABELLED SOLID MEALS Pancakes of differing consistency were made from plain flour $(100 \mathrm{~g})$, milk $(200 \mathrm{ml})$, baking powder $(4 \mathrm{~g})$, salt $(0.5 \mathrm{~g})$ and one egg. In the first recipe, the ingredients were stirred together, resulting in pancakes of a dense consistency. In the second recipe, Russian pancakes of a light and fluffy consistency were made by using heated milk and whisking the egg white. One hundred microcuries ${ }^{4 m}$ Technetium sulphur colloid (CIS (UK) Ltd, London, UK) was added to $50 \mathrm{ml}$ of the pancake mixture before cooking.

IN Vitro STUDiEs

Studies were conducted to investigate the rates of breakdown of the two types of pancake during mechanical agitation in an acid pepsin or saline medium.

Ten grams of cooked radiolabelled pancake was chopped into $1 \mathrm{~cm}^{3}$ pieces and placed in stainless steel wire mesh baskets measuring $2.5 \mathrm{~cm} \times 2.5 \mathrm{~cm} \times$ 
$5 \mathrm{~cm}$ with a mesh size of $2 \mathrm{~mm}$. The baskets were suspended in flasks containing $150 \mathrm{ml}$ of either gastric juice at $\mathrm{pH} 1$ or normal saline. The gastric juice had previously been aspirated from human subjects after administration of a subcutaneous injection of pentagastrin (Peptavlon, $6 \mu \mathrm{g} / \mathrm{kg}$ bodyweight) and was made up to $\mathrm{pH} 1$ by addition of concentrated hydrochloric acid. The flasks were fixed in a water bath maintained at $37^{\circ} \mathrm{C}$ and shaken at $110-120$ cycles per minute for a period of three hours. Five millilitre aliquots of bathing medium was removed at 30 minute intervals and the radioactivity in each sample was determined using a single crystal scintillation counter linked to a counter ratemeter (Scintillation Counter DM1-2, Nuclear Enterprises Ltd, Edinburgh). After each sample had been taken the volume of the bathing medium was made up with the appropriate medium. After 180 minutes the entire contents of the flask were homogenised and a $5 \mathrm{ml}$ sample counted.

\section{ANALYSIS OF IN VITRO STUDIES}

The following equation was used to correct the number of counts in each sample for the serial dilutions to yield a value for the number of counts in the bathing medium at each time period.

$$
C_{150}=C_{5} \times\left(\frac{150}{145}\right)^{n} \times \frac{150}{5}
$$

where ' $\mathrm{C}_{150}$ ' is the counts in $150 \mathrm{ml}$ bathing medium for each time period; ' $C_{5}$ ' is the number of counts per minute in the $5 \mathrm{ml}$ sample, corrected for background radiation and isotope decay and ' $n$ ' is the number of dilutions.

The percentage of the radioactivity remaining within the basket at 5 minute intervals was calculated and plotted against time.

\section{IN VIVO STUDIES}

Subjects

Studies were carried out in one female and eight male healthy volunteers, who were aged between 18 and 26 years. All subjects gave their fully informed consent for the study to be carried out. The female volunteer signed a statement to the effect that she was taking a reliable form of contraception and that, to her knowledge, she was not pregnant.

The equipment used conformed to the safety standards laid down by the Department of Health and the study was carried out in accordance with the Declaration of Helsinki and with the approval of the Ethical Sub-Committee of the Sheffield Southern District Hospital.

\section{Experimental Protocol}

Six subjects were studied on two separate occasions separated by periods of at least three days. On each occasion, they were given either the radiolabelled light consistency pancake (Blini) or the dense consistency pancake (traditional); the order of the studies was randomised.

The subjects fasted for at least 12 hours before each study. No alcohol was permitted for 24 hours before each study and cigarette smoking was not allowed. On the day of the study, the subject sat at a $45^{\circ}$ angle in a dental chair and a gamma camera (Model 1201 Pho/Gamma Scintillation Camera, Nuclear-Chicago, Europa NV, Amsterdam, The Netherlands) was positioned over the abdomen. The subject was then given the radiolabelled pancake meal, which had previously been chopped into $1 \mathrm{~cm}^{3}$ pieces, and was instructed to swallow the pieces of pancake without chewing. Five minutes were allowed for subjects to ingest the meal. To aid ingestion of the meals, the subjects were permitted to take sips of water up to a total volume of $50 \mathrm{ml}$.

The gamma camera was linked to a dedicated mini computer which was programmed to record radioactive counts from the stomach over consecutive five minute periods for a total of 120 minutes. Data collection began at the beginning of food ingestion.

The paired studies were repeated in the same six volunteers and in a further three volunteers after a subcutaneous injection of pentagastrin (Peptavlon, $6 \mu \mathrm{g} / \mathrm{kg}$ bodyweight), which was given 15 minutes before the start of the study.

ANALYSIS OF GAMMA CAMERA RECORDS

The position of the stomach was identified from an integrated image of the first two frames and outlined using a cursor. The study was then played back to confirm that the stomach remained within the outlined region of interest throughout the study. The computer then extracted the counts from the region of interest, corrected them for isotope decay and expressed them as a percentage of the counts obtained in the gastric region immediately after ingestion of the meal. These figures were then used to construct a profile of the proportion of counts in the whole stomach throughout the study, from which the times taken for $10,25,40,50\left(t_{1 / 2}\right)$ and $70 \%$ of the meal to empty from the stomach and the rate of linear emptying were obtained.

The time from the end of ingestion to the time at which the meal started to empty was known as the lag period and was obtained by measuring the time that the marker first appeared in a region of interest, that comprised all the field outside the gastric region.

STATISTICAL ANALYSIS

Data were analysed using the Student's paired or unpaired $t$ test as appropriate. 


\section{Results}

IN VITRO STUDIES

Figure 1 shows that less than $3 \%$ of radioactivity appeared in the medium when either pancake was subjected to mechanical agitation in normal saline for 150 minutes. The rate of appearance of marker was much greater in the presence of acid pepsin compared with normal saline (Fig. 1). Under acid conditions, radioactivity from the lighter pancake accumulated more rapidly in the medium than that from the dense pancake $(0.5 \pm 0.1 v 0.2 \pm 0.1 \%$ of radioactivity appearing in the bathing medium per minute, $\mathrm{p}<0 \cdot 01$ ) (Fig. 1). After 150 minutes, $92 \%$ of the radioactivity from the lighter pancake had been released into the medium compared with only about $26 \%$ of the marker from the denser pancake $(\mathrm{p}<0 \cdot 01)$. Thus the light consistency (Blini) pancake was disrupted more quickly than the dense consistency (traditional) pancake in acid pepsin medium.

IN VIVO STUDIES

Gastric emptying of the pancake meals under normal conditions

After an initial lag period, the pancakes emptied

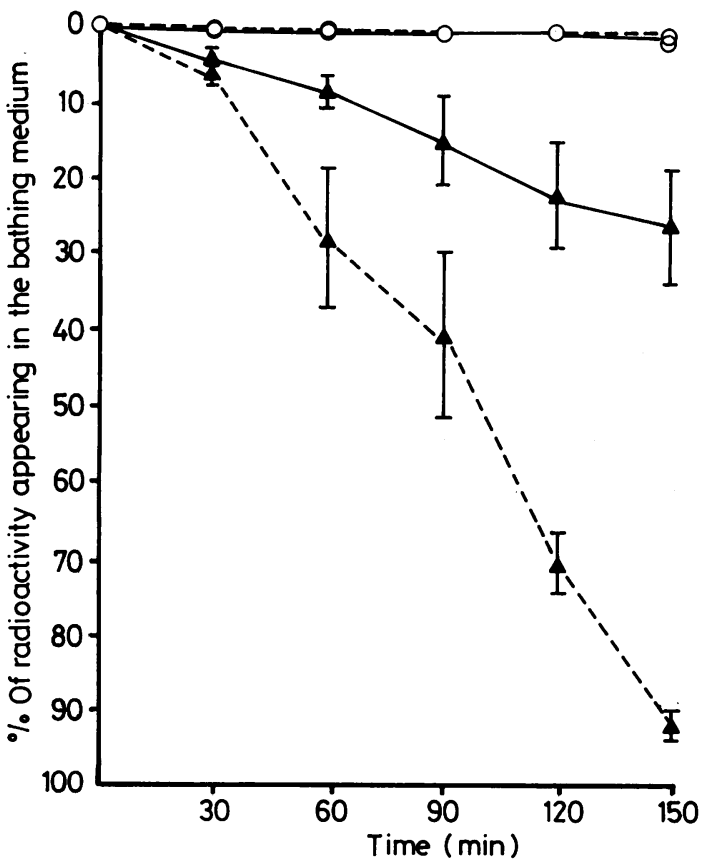

Fig. 1 Comparison of the rate of appearance of radioactivity in the medium, when the light consistency (Blini) pancake (-----) and the dense consistency (traditional) pancake (-) when subjected to mechanical agitation in a solution of either normal saline $(\bigcirc)$ or acid-pepsin $\mathrm{pH} 1(\mathbf{\Delta})$. linearly from the stomach (Fig. 2a). The lag periods and the slopes of emptying of the two types of pancake meal were very similar (Fig. 2a) (Table 1).

\section{Gastric emptying of the pancake meals after a} subcutaneous injection of pentagastrin

After administration of pentagastrin, the lag periods for the emptying of the dense $(p<0 \cdot 01)$ and light $(p<0 \cdot 05)$ pancake meals were significantly prolonged and the slope of the emptying phase was accelerated, though the latter did not achieve statistical significance for either meal (Table 2) (Fig. 3). This meant that the times taken for $10 \%(p<0.01)$ and $25 \%$ $(p<0.05)$ of the dense pancake and $10 \%(p<0.05)$ of the light pancake were longer after administration of pentagastrin whereas there were no differences in the times taken for 40,50 , and $70 \%$ of the dense pancake and for $25,40,50$, and $70 \%$ of the light pancake to empty from the stomach under normal conditions compared with pentagastrin stimulated conditions.

After administration of pentagastrin, the times taken for $10,25,40$, and $50 \%$ of the denser pancake to empty from the stomach were significantly longer compared with the light (Blini) pancake $(\mathrm{p}<0.05)$ (Fig. 2b). The delay in emptying of the dense pancake was associated with a longer lag period $(p<0 \cdot 01)$, but no difference in the slope of the emptying phase (Table 1, Fig. 2b).

\section{Discussion}

The studies that were carried out in vitro showed that neither of the pancake meals were broken up within 150 minutes by mechanical agitation in the normal saline medium, but both were disrupted in the presence of acid pepsin. This illustrates the role of acid digestion in the disruption of solid food. The observation that the Blini pancake was disrupted by mechanical agitation in the presence of acid pepsin much more quickly than the traditional pancake is compatible with its less dense consistency, which would allow more rapid penetration of acid pepsin and would present a larger surface area for peptic digestion. Thus the in vitro data would predict that the more readily disrupted Blini pancake would empty more rapidly from the stomach.

Measurements of gastric emptying in normal volunteers did not altogether confirm the predictions of the in vitro studies; the pattern and rate of gastric emptying of both pancake meals were not significantly different under normal conditions of acid secretion. One interpretation of these results is that the compression and rapid accelerations and decelerations achieved by antral contractions disrupt the pancakes so rapidly in vivo that emptying of these pancake meals is limited more by factors that 

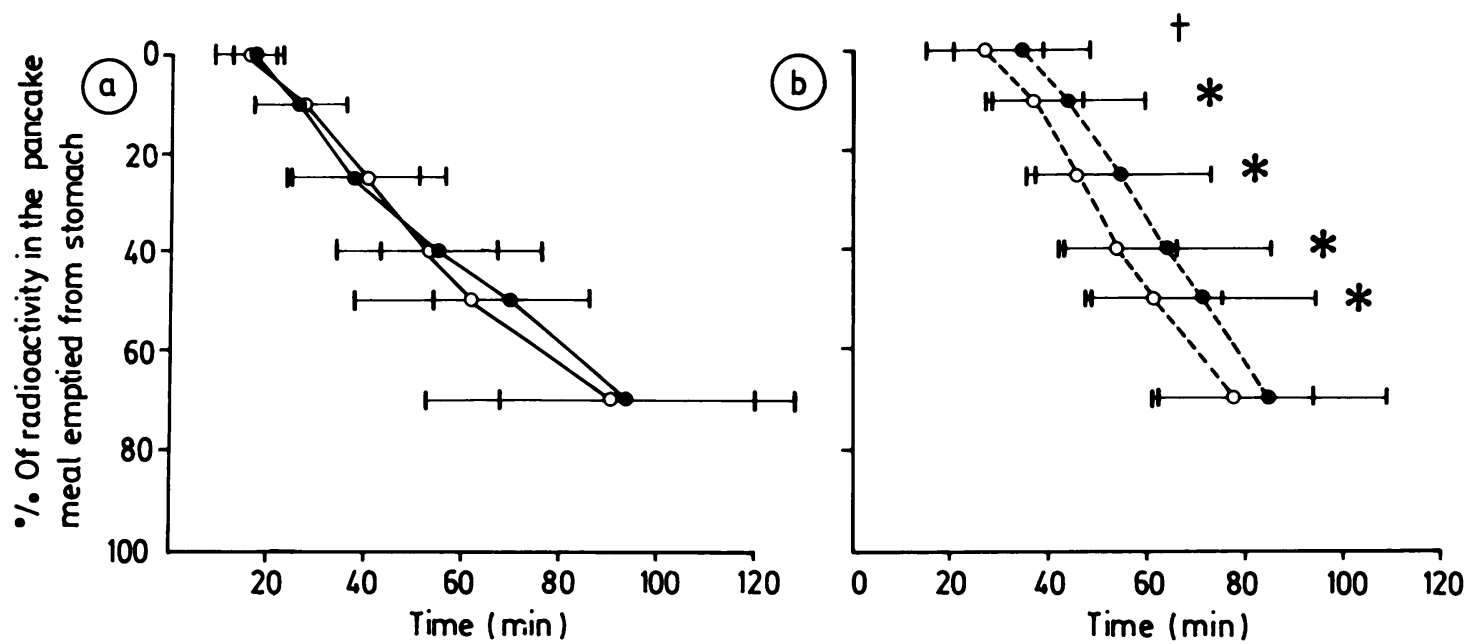

Fig. 2 Comparison of the times taken for the radioactive label in the dense $(O)$ and light (O) consistency pancakes to empty from the stomach, under normal conditions (a) or after a subcutaneous injection of pentagastrin ( $6 \mu \mathrm{g} / \mathrm{kg}$ bodyweight) (b). $\operatorname{Mean}(S D) ;{ }^{*} p<0 \cdot 05,{ }^{+} p<0 \cdot 01$.

regulate gastric contractility than by differences in disruptibility. Alternatively, it is possible that the pancake meals did not stimulate sufficient gastric acid secretion to cause a difference in disruption in vivo or that inhibition of gastric motility caused by the presence of acid in the duodenum masked the effects of peptic digestion on gastric emptying. ${ }^{\text {? }}$

To investigate these possibilities, we carried out similar studies after stimulation of acid-pepsin secretion by administration of pentagastrin. Ad-

Table 1 Comparison of indices of gastric emptying of dense consistency (traditional) pancake and light consistency (Blini) pancake labelled with ${ }^{4 * m}$ Tc-sulphur colloid under normal conditions and after a subcutaneous injection of pentagastrin ( $6 \mu \mathrm{g} / \mathrm{kg}$ bodyweight)

\begin{tabular}{|c|c|c|c|}
\hline & $\begin{array}{c}\text { Dense } \\
\text { consistency } \\
\text { (traditional) } \\
\text { pancake }\end{array}$ & $\begin{array}{c}\text { Light } \\
\text { consistency } \\
\text { (Blini) } \\
\text { pancake }\end{array}$ & $p$ \\
\hline \multicolumn{4}{|l|}{ Under normal conditions } \\
\hline Number of subjects & 6 & 6 & \\
\hline Half time $t_{1},(\min )$ & $70 \cdot 0 \pm 16 \cdot 3$ & $62 \cdot 0 \pm 24 \cdot 1$ & NS \\
\hline Lag period (min) & $16 \cdot 7 \pm 4 \cdot 6$ & $15 \cdot 8 \pm 7 \cdot 2$ & NS \\
\hline $\begin{array}{l}\text { Slope ( } \% \text { emptied } \\
\text { per minute) }\end{array}$ & $1 \cdot 2 \pm 0.9$ & $1 \cdot 3 \pm 0 \cdot 6$ & NS \\
\hline \multicolumn{4}{|c|}{ After pentagastrin administration } \\
\hline Number of subjects & 9 & 9 & \\
\hline Half time $t_{1_{2}}(\min )$ & $71 \cdot 6 \pm 23 \cdot 4$ & $61 \cdot 4 \pm 14 \cdot 0$ & $<0.05$ \\
\hline Lag period $(\min )$ & $34 \cdot 5 \pm 14 \cdot 1$ & $26 \cdot 6 \pm 11 \cdot 8$ & $<0 \cdot 01$ \\
\hline $\begin{array}{l}\text { Slope ( } \% \text { emptied } \\
\text { per minute) }\end{array}$ & $1 \cdot 6 \pm 0 \cdot 6$ & $1 \cdot 7 \pm 0.8$ & NS \\
\hline
\end{tabular}

Results are mean \pm SD. ministration of pentagastrin more than doubled the lag periods for emptying of both pancake meals, but tended to accelerate the rate of emptying. It is likely that the delay in onset of solid emptying is related to the interaction of excess gastric acid with duodenal receptors. Infusion of acid into the duodenum is known to decrease fundal contraction, suppress antral contractility and increase pyloric resistance. Moreoever, exposure of the duodenal receptors to liquids known to delay gastric emptying, such as hyperosmotic fluids, or lipids increase the lag period, but do not alter the rate at which solids leave the

Table 2 Effect of administration of pentagastrin $(6 \mu \mathrm{g} / \mathrm{kg}$ bodyweight) on the indices of gastric emptying of dense consistency (traditional) pancake and light consistency (Blini) pancake labelled with ${ }^{4 m_{m}} T$-sulphur colloid

\begin{tabular}{|c|c|c|c|}
\hline & $\begin{array}{c}\text { Normal } \\
\text { conditions } \\
(n=6)\end{array}$ & $\begin{array}{c}\text { After } \\
\text { administration of } \\
\text { pentagastrin } \\
(6 \mu g / k g \\
\text { bodyweight }) \\
(n=9)\end{array}$ & $p$ \\
\hline \multicolumn{4}{|c|}{ Dense consistency (traditional) pancake } \\
\hline Lag time $(\mathrm{min})$ & $16 \cdot 7 \pm 4 \cdot 6$ & $34 \cdot 5 \pm 14 \cdot 1$ & $<() \cdot 01$ \\
\hline $\begin{array}{l}\text { Slope ( } \% \text { emptied } \\
\text { per minute) }\end{array}$ & $1.2 \pm 0.9$ & $1.6 \pm 0.6$ & NS \\
\hline \multicolumn{4}{|c|}{ Light consistency (Blini) pancake } \\
\hline Lag time (min) & $15 \cdot 8 \pm 7 \cdot 2$ & $36 \cdot 6 \pm 11 \cdot 8$ & $<0 \cdot 05$ \\
\hline $\begin{array}{l}\text { Slope ( } \% \text { empticd } \\
\text { per minute) }\end{array}$ & $1 \cdot 3 \pm 0 \cdot 6$ & $1.7 \pm 0.8$ & NS \\
\hline
\end{tabular}

Results are mean \pm SD. 

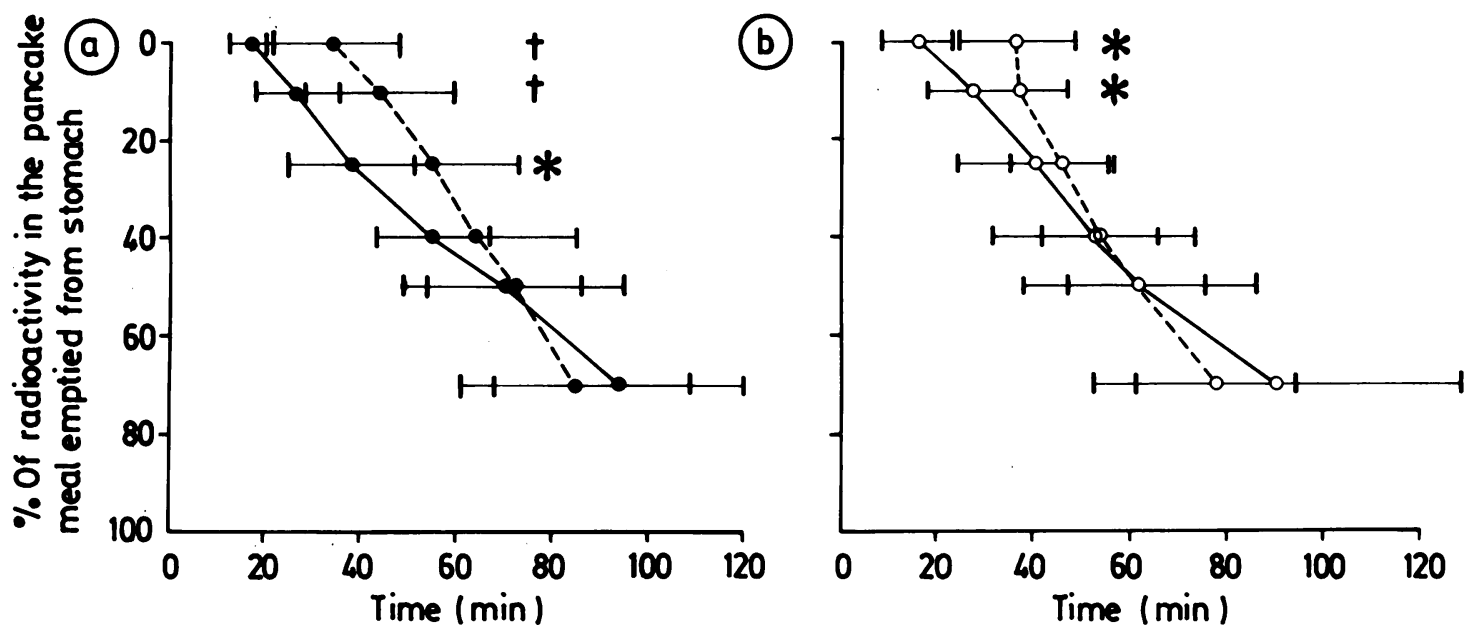

Fig. 3 Comparison of times taken for the radioactive label in the dense $(a)$ and light $(b)$ consistency pancake to empty from the stomach after a subcutaneous injection of pentagastrin $(6 \mu \mathrm{g} / \mathrm{kg}$ bodyweight) (-----) compared with normal conditions (-). Administration of pentagastrin, prolonged the lag periods and accelerated the rate of emptying of both pancake meals. Mean $(S D) ;{ }^{*} p<0.05 ; \dagger p<0 \cdot 01$.

stomach. ${ }^{8-11}$ The trend towards acceleration in the rate of emptying is compatible with the increased breakdown of the meal in the presence of surplus acid pepsin as indicated by the in vitro studies.

After administration of pentagastrin, the traditional pancake emptied more slowly than the Blini pancake, supporting the suggestion from the in vitro studies that the acid-pepsin penetrates and breaks down less dense food more easily than dense food. This should result in an alteration of the slope of emptying, however, rather than an increase in the lag phase. The observation that the lag phase was increased and not the slope is perhaps best explained by the probability that the dense traditional pancake would have less protein groups accessible to buffer hydrogen ions than the Blini pancake and thus allow more acid to interact with duodenal receptors.

In conclusion, although our data offer some support for the hypothesis that the rate of emptying of disruptible solid food may be limited by the ease at which it can be broken down in a gastric environment, other factors, such as suppression of gastric contractions by free hydrogen ions must also have an important influence. Under normal circumstances, the emptying of pancake meals are limited more by gastric propulsion than by the mechanical disruption, but this conclusion may not necessarily apply to foods that are more difficult to break up, such as meat.

This work was supported by a grant from Smith, Kline and French Research Limited.

\section{References}

1 Kelly KA. Gastric emptying of liquids and solids: roles of proximal and distal stomach. Am J Physiol 1980; 239: G71-6.

2 Hinder RA, Kelly KA. Canine gastric emptying of solids and liquids. Am J Physiol 1977; 233: E335-40.

3 Meyer JH, MacGregor IL, Gueller R, Martin P, Cavalieri R. ${ }^{{ }^{9} \mathrm{~m}} \mathrm{Tc}$-tagged chicken liver as a marker of solid food in the human stomach. Dig Dis Sci 1976; 21: 296-304.

4 Meyer JH, Thomson JB, Cohen MB, Shadchehr A, Mandiola SA. Sieving of solid food by the canine stomach and sieving after gastric surgery. Gastroenterology 1979; 76: 804-13.

5 Holt S, Reid J, Taylor TV, Tothill P, Heading RC. Gastric emptying of solids in man. Gut 1982; 23: 292-6.

6 Weiner K, Graham LS, Reedy T, Elashoff J, Meyer JH. Simultaneous gastric emptying of two solid foods. Gastroenterology 1981; 81: 257-66.

7 Ohashi H, Meyer JH. Effect of peptic digestion on emptying of cooked liver in dogs. Gastroenterology 1980; 79: 305-10.

8 Collins PJ, Horowitz M, Cook DJ, Harding PE, Shearman DJC. Gastric emptying in normal subjects - a reproducible technique using a single scintillation camera and computer system. Gut 1983; 24: 1117-25.

9 Houghton LA, Read NW, Heddle R, Horowitz M, Collins PJ, Dent J. The relationship between gastric emptying and the motor activity of the antrum, pylorus and duodenum after ingestion of a solid-liquid mixed meal [Abstract]. Gastroenterology 1986; 90: 1465.

10 Collins PJ, Heddle R, Horowitz M, Read NW, Dent J, Chatterton BE. The effect of intraduodenal lipid on gastric emptying and intragastric distribution of a solid meal [Abstract]. Gastroenterology 1986; 90: 1377. 